\section{EL SUICIDIO EN} ADOLESCENTES: UN ANÁLISIS DESDE LAS DESVENTAJAS SOCIALES - ECONÓMICAS Y LAS ESTRATEGIAS DE REGULACIÓN EMOCIONAL

Yuly Paola Suárez-Colorado Universidad Cooperativa de Colombia

El presente documento de trabajo ha sido incluido dentro de nuestro repositorio institucional como Apropiación social de conocimiento por solicitud del autor, con fines informativos, educativos o académicos. Asimismo, los argumentos, datos y análisis incluidos en el texto son responsabilidad absoluta del autor y no representan la opinión del Fondo Editorial o de la Universidad.

\title{
DISCLAIMER
}

This coursework paper has been uploaded to our institutional repository as Social Appropriation of Knowledge due to the request of the author. This document should be used for informational, educational or academic purposes only. Arguments, data and analysis included in this document represent authors' opinion not the Press or the University.

Apropiación social del conocimiento Generación de contenidos impresos https://repository.ucc.edu.co/handle/20.500.12494/7375 N. ${ }^{\circ} 05$, febrero de 2020 doi: https://doi.org/10.16925/gclc.09 


\section{ACERCA DE LA AUTORA}

Yuly Paola Suárez-Colorado. Magíster en Psicología. Profesora instructora de la Facultad de Psicología, Universidad Cooperativa de Colombia, sede Santa Marta. Coordinadora de Posgrados de la Facultad de Psicología.

Correo-e: yuli.suarezcol@campusucc.edu.co CvLAC: https://scienti.colciencias.gov.co/cvlac/visualizador/generarCurriculoCv.do?cod_rh=0001404153

ORCID: http://orcid.org/0000-0001-7715-3556

Google Scholar: https://scholar.google.es/citations?user $=$ mRUw4xIAAAAJ\&hl=es

Research Gate: https://www.researchgate.net/profile/Yuly_Suarez-Colorado

\section{CÓMO CITAR ESTE DOCUMENTO}

Suárez-Colorado, Y. P. (2020). El suicidio en adolescentes: un análisis desde las desventajas sociales - económicas y las estrategias de regulación emocional (Generación de contenidos impresos N.5). Bogotá: Ediciones Universidad Cooperativa de Colombia. doi: https://doi. org/10.16925/gclc.09

Este documento puede ser consultado, descargado o reproducido desde nuestro repositorio institucional (http://repository.ucc.edu.co/ handle/20.500.12494/7369) para uso de sus contenidos, bajo la licencia de Creative Commons Reconocimiento-NoComercial-SinObraDerivada 4.0 Internacional. http://creativecommons.org/licenses/by-ncnd/4.0/ 


\title{
05 EL SUICIDIO EN ADOLESCENTES: UN ANÁLISIS DESDE LAS DESVENTAJAS SOCIALES - ECONÓMICAS Y LAS ESTRATEGIAS DE REGULACIÓN EMOCIONAL
}

\author{
Yuly Paola Suárez-Colorado
}

\section{Resumen}

El suicido es un problema de salud pública mundial con tendencia a incrementarse en población cada vez más joven; así mismo, las estadísticas mundiales señalan un comportamiento particular de acuerdo con las condiciones sociales y económicas. Las desventajas socioeconómicas originarias de la violencia estructural consisten en un conjunto de situaciones de exclusión y violación de derechos humanos, sociales, económicos y culturales, que determinan la supervivencia, y algunos adolescentes inmersos en estos contextos deben además enfrentar situaciones adversas que demandan del despliegue de estrategias exitosas de regulación emocional. No obstante, no todos los adolescentes pueden acceder a estrategias efectivas y por el contrario el uso de estrategias no efectivas puede deteriorar la salud mental a largo plazo y propiciar conductas de riesgo como el suicidio. En esta lectura crítica se presenta el análisis de la adolescencia como etapa de transición, la desventaja social-económica desde la violencia estructural, la conducta suicida desde las desventajas sociales-económicas, la regulación emocional desde Gross, la regulación emocional en contextos de desventaja social-económica y de forma específica la regulación emocional y la conducta suicida. Finalmente, se aportan algunos fragmentos que demuestran la naturaleza de las necesidades psicoafectivas de los adolescentes. Esta lectura crítica es una aproximación parcial a un problema multifactorial como el suicidio.

Palabras clave: adolescentes, desventajas sociales y económicas, regulación emocional, suicidio. 


\section{TABLA DE CONTENIDO}

1. INTRODUCCIÓN

2. Desarrollo del contenido 5

2.1 La adolescencia: una etapa de transición 5

2.2 La desventaja social y económica desde la violencia estructural 6

2.3 Análisis de la conducta suicida desde las desventajas 7 sociales y económicas

2.4 Vislumbrar la regulación emocional desde Gross 11

2.5 La regulación emocional en contextos de desventaja 13 social y económica

2.6 Otras estrategias para la gestión de emociones 13

2.7 La regulación emocional y la conducta suicida 15

2.8 Identificación de las necesidades psicoafectivas de los adolescentes 16

3. Eíĺlogo 17

$\begin{array}{ll}\text { REFERENCIAS } & 21\end{array}$ 


\section{INTRODUCCIÓN}

El suicidio en adolescentes es un importante problema de salud pública sin determinantes exclusivos, aunque en algunas ocasiones se manifiesta ante las múltiples experiencias adversas. La comprensión del suicidio en adolescentes puede contribuir al diseño e implementación de estrategias de prevención efectivas; por esta razón, es relevante reconocer la interacción de factores sociales, económicos y psicológicos.

Diversos estudios exploran el vínculo entre las condiciones sociales y económicas asociadas a los comportamientos suicidas; y de forma particular, cómo la vulnerabilidad, la desintegración y la exclusión social afectan la salud, la calidad de vida y el desarrollo humano. Por otra parte, la gestión emocional implica un esfuerzo cognitivo, afectivo y conductual para las diferentes etapas del desarrollo, porque no es natural en los seres humanos desplegar procesos de regulación emocional, especialmente cuando no es el primer eslabón de las competencias emocionales. La regulación emocional es un proceso complejo y especializado que implica estrategias específicas de acuerdo con la instrucción, el evento de exposición y los recursos sociales, económicos o afectivos del contexto.

Esta lectura crítica busca analizar el riesgo suicida en la adolescencia desde la desventaja social y económica, así como las estrategias de regulación emocional. El objetivo es aproximar al lector a las particularidades de las estrategias de regulación emocional en la adolescencia en contextos afectados por la violencia estructural, y cómo las estrategias inadecuadas están asociadas al riesgo de suicidio en una etapa de transición vital como la adolescencia.

Esta temática es relevante en el área del desarrollo integral de niños y adolescentes para el diseño de proyectos de investigación básica y aplicada que reconozcan las necesidades de salud y los recursos psicológicos de las poblaciones vulnerables. La lectura está conformada por una aproximación a la adolescencia como etapa de transición; la comprensión de las desventajas sociales y económicas a través de la violencia estructural; el análisis de la conducta suicida desde los indicadores económicos que instauran las desventajas socioeconómicas; la contextualización teórica de la regulación emocional; y la particularidad de las estrategias de regulación emocional en contextos vulnerables.

Finalmente, este material permite considerar que las estrategias de prevención del suicidio basadas en el fomento de recursos psicológicos como la regulación emocional deben adecuarse a las condiciones sociales y económicas del contexto. En consecuencia, los proyectos deberán incluir las estrategias más efectivas para la salud en poblaciones vulnerables, teniendo como base la educación en gestión de emociones recibida y las respuestas de regulación más adaptativas. A partir de esta base, los proyectos deben incorporar estrategias de intervención desde las posibilidades de potenciar recursos en la población.

\section{Desarrollo del contenido}

\subsection{LA ADOLESCENCIA: UNA ETAPA DE TRANSICIÓN}

La adolescencia es una etapa de transición del desarrollo humano caracterizada por la presencia de algunos rasgos de la infancia y el deseo de aspectos de la etapa adulta. Es una etapa de transición en la medida en que se produce un crecimiento cognitivo, emocional, social y físico en medio de la crisis; la experiencia de la crisis adolescencial se convierte en una oportunidad para la construcción de la identidad, los valores, los significados y los recursos psicológicos.

Se espera que el desarrollo cognitivo del adolescente se consolide en madurez cognitiva 
para la resolución de problemas desde el razonamiento lógico; no obstante, gran parte de la población se ubica en etapas transitorias. El desarrollo social interactúa con diferentes factores socioecológicos que conforman varias dinámicas relacionales en la adolescencia. Mientras, el desarrollo emocional se trastoca por las transformaciones de los roles en sociedad, la competitividad y los avances tecnológicos que instauran diversas formas de vínculos psicoafectivos entre padres-hijos y pares-pares. Adicionalmente, el desarrollo físico se percibe de acuerdo con los cambios sociales y económicos globales que también son transitorios. En general, la perspectiva del desarrollo del adolescente también es transitoria dados los avances de la sociedad en ciencia, tecnología e innovación, y por consiguiente, en la medición y evaluación del desarrollo de acuerdo con el momento histórico.

En este sentido, la adolescencia debe ser vista como una etapa de transición y no de crisis, aun cuando el desarrollo supone un proceso no lineal de adquisición y pérdidas; finalmente, el adolescente adquiere y pierde para su crecimiento cognitivo, social, emocional y físico.

\subsection{LA DESVENTAJA SOCIAL Y ECONÓMICA DESDE LA VIOLENCIA ESTRUCTURAL}

Raymond Aron (1980, p. 45), conocedor de la obra de Durkheim, expresó "lo que le interesa por encima de todo, hasta el punto de obsesionarlo, es, en efecto, la crisis de la sociedad moderna que se define por la desintegración social y la debilidad de los vínculos que relacionan al individuo con el grupo". Algunos factores de riesgo para el comportamiento suicida se vinculan directa o indirectamente a la exclusión y vulnerabilidad que conducen sistemáticamente a la desintegración, propia de la violencia estructural o violencia colectiva.

De acuerdo con Parra y Tortosa (2003), esta forma de violencia corresponde a las necesidades básicas insatisfechas relacionadas con el bienestar, la identidad, la libertad y la supervivencia producto de la estratificación social. Al mismo tiempo, puede vincularse con manifestaciones de violencia directa (cambio de posición social por la fuerza) o de violencia cultural (legitimizaciones de otras formas de violencia).

Para comprender un poco más acerca de la violencia estructural, las siguientes palabras de Engels nos sitúan en una forma de violencia indirecta, invisible, manifiesta por un agresor-víctima de difícil identificación, así como los obstáculos para determinar los mecanismos explicativos:

Cuando la sociedad sitúa a cientos de proletarios en una posición en la que de forma inevitable se encontrarán con una muerte prematura e inevitable [...], cuando priva a miles de personas de la satisfacción de las necesidades vitales, situándolos en condiciones en las que no es posible vivir —obligándolos, a través de la fuerza de la ley, a permanecer en esas condiciones hasta que la muerte sea la consecuencia inevitable-, la sociedad sabe que esos miles de víctimas perecerán y aun así permite que esas condiciones se mantengan, este acto es un asesinato [...] asesinato disfrazado e intencionado contra el que nadie puede defenderse por sí mismo [...] porque nadie ve al asesino, porque la muerte de la víctima parece natural en tanto que el delito es más por omisión que por comisión. Pero asesinato, al fin y al cabo. (Engels, 1844, citado en Parra y Tortosa, 2003, p. 59)

La violencia estructural es un concepto equivalente a pobreza, injusticia social, desigualdad y exclusión social (Galtung, 1985). Rodríguez (2004) plantea específicamente que la pobreza está vinculada con: a) situaciones de exclusión, relacionadas con la falta de acceso 
a canales de comunicación, la ausencia de espacios de participación que permitan tomar decisiones sobre la comunidad-sociedad; y b) violación de derechos humanos, fundamentada en la carencia de recursos materiales. Por consiguiente, corresponde a la insatisfacción de necesidades básicas y privación de capacidades que truncan el desarrollo.

Una aproximación sintética a estos dos componentes de la violencia estructural reafirma que esta forma de violencia se fundamenta en:

Exclusión/participación social. Inicialmente, los procesos de exclusión se analizan desde dos perspectivas: la personal y la global; es decir, existen dos vertientes de exclusión entre ellas, el individuo que propiamente se excluye de la participación o el sistema que es excluyente (Tezanos, 2001). Pero no solo involucra el problema de la desigualdad, sino también las distancias en la estructura social (Consejo Económico y Social, 1997). Así mismo, algunos autores han relacionado directamente la exclusión con la privación del goce de derechos fundamentales, pero sin duda emerge más allá de las privaciones económicas (Sen, 2008). Subirats (2004) establece los marcos de participación sobre la cual se fundamenta la exclusión: el mercado y/o la utilidad social personal como herramienta de intercambio-aporte a la colectividad, redistribución de connotación administrativa-pública, y las relaciones de reciprocidad en las redes sociales-familiares.

Violación de derechos humanos sociales, económicos, culturales. Los derechos al trabajo, la seguridad social, la vida en familia, la participación en la vida cultural, el acceso a la vivienda, la alimentación, el agua, la atención en salud y la educación conforman el banco de derechos sociales, económicos y culturales (Oficina del Alto Comisionado de las Naciones Unidas para los Derechos Humanos, 2009). Básicamente, estos derechos garantizan la satisfacción de las necesidades básicas para ejercer la autonomía-libertad y pueden en general discriminarse así (Comisión Económica para América Latina y el Caribe [Cepal], 2009): derechos económicos, como alimentación vivienda, trabajo e ingresos; derechos sociales, referentes a la seguridad social, salud física y mental; y derechos culturales, concernientes a la educación y a la información, entre otros derechos de los grupos minoritarios.

En Colombia los derechos sociales, económicos y culturales se fundamentan en la Constitución de 1991, y en síntesis, se soportan en el artículo 44, aunque pueden involucrar otros artículos: el derecho a la integridad física, a la salud y a la seguridad social, a la alimentación, al nombre, a la nacionalidad, a la familia y no ser separados de ella, al cuidado-amor, a la educación, a la cultura, a la recreación y a la libre expresión. De manera que la violación de estos derechos representa la vulnerabilidad por desventaja social y económica.

\subsection{ANÁLISIS DE LA CONDUCTA SUICIDA DESDE LAS DESVENTAJAS SOCIALES Y ECONÓMICAS}

El suicidio es un problema global que anualmente genera un millón de muertes en el mundo, es decir, dieciséis muertes por cada 100000 habitantes. El incremento de casos en los últimos 45 años, representado en $60 \%$, se acentúa en personas entre 15 y 44 años (Organización Mundial de la Salud [OMs], 2012). El pronóstico para el 2020 es del 2,4 \% en la carga de morbilidad global por suicidio en países con economías de mercado y en los antiguos países socialistas.

Es un problema de relevancia estadística mundial, aunque las cifras excluyan los intentos de suicidio, veinte veces más frecuentes que las muertes por suicidios (OMs, 2012). Los registros de suicidio en el mundo señalan, en primer lugar, a Guyana, seguido de República de Corea y China. Otra zona de importante prevalencia de suicidio consumado se observa en África con una tasa de 11,4 por 
cada 100000 habitantes, y en comparación con el año 2000 hay un aumento del 38 \%. En América, la tasa suele ser más baja; no obstante, Guyana tuvo la tasa más alta en todo el mundo y Suriname ocupó el sexto lugar. En el Mediterráneo Oriental, la tasa es más baja. Europa evidenció una tasa de 11,4 por cada 100000 habitantes, y veinte países europeos presentaron las tasas más altas en el mundo; por ejemplo, Lituania se situó en quinto lugar y Kazajstán, en el décimo. Asia Suroriental evidenció la concentración de mayores casos de suicidio en el mundo con India. En el Pacífico Occidental, la tasa es inferior al 11,4, que es la media mundial. Sin embargo, República de Corea se destaca como el tercer país con la tasa más alta del mundo, y China se ubicó en el segundo lugar (OMS, 2014).

En el caso de Colombia, las muertes con causa no especificada son alarmantes y en la última década se registraron 20832 casos de suicidio, con promedio anual de 2083 casos (Instituto Colombiano de Medicina Legal y Ciencias Forenses, 2019). Para el 2018, el Instituto de Medicina Legal realizó 2696 necropsias debidas a suicidio, lo que indica una mortalidad por suicidios consumados de 5,93 casos por cada 100000 habitantes. La tasa nacional para el rango de 15-17 años se encontró en 6,64 por cada 100000 habitantes. Sin embargo, el mayor número de casos estuvo en el rango de 20-24 años con una tasa de 9,09 por cada 100000 habitantes (Instituto Colombiano de Medicina Legal y Ciencias Forenses, 2015). Las razones suicidas registradas en los casos nacionales son los conflictos interpersonales, rupturas afectivas, enfermedad física-mental, muerte de un ser querido, dificultades escolares, víctima de maltrato físico-sexual-psicológico, antecedente suicidio familiar, dificultades económicas, bullying, etc.

En relación con las condiciones sociales-económicas de los contextos, existen serios cuestionamientos acerca de la efectividad de las políticas de prevención en los países. La base de estos cuestionamientos es la explicación de la problemática desde un enfoque de vulnerabilidad, es decir, los contextos donde mayormente surgen decesos por suicidios carecen de recursos psicosociales para sostener la vida de la población. En este sentido, es posible plantear que el suicidio es un problema ante todo social que destella la fragilidad de un ser humano inmerso en una sociedad que ejerce violencia invisible (la estructural) en su contra, al mantener la vulnerabilidad, la exclusión y la desintegración desatendiendo las necesidades básicas humanas.

El Instituto de Medicina Legal y Ciencias Forenses (2015, p. 323) indica que:

[...] aunque el suicidio ha existido en todas las épocas, esta problemática establece a la sociedad tres interrogantes: ¿es un acto de cobardía, temor, pánico, desesperanza?, ¿o quizá un acto de coraje, valentía o de bravura? Pero por qué no plantear un tercer interrogante: ¿es un acto de falta de oportunidad?, ¿oportunidad de qué? De ser escuchado, de ser percibido, de ser atendido, de sentirse amado.

Este tercer interrogante atiende a los postulados de Durkheim (1897), quien considera que el suicidio varía según la desintegración social de la sociedad, o religiosa, doméstica o política, pero además que puede surgir a razón de factores económicos, climáticos, geográficos, la guerra, la religión, etc. Es un problema de salud, pero también es un problema social; por tanto, el riesgo de suicidio puede estar presente en personas con algún factor de vulnerabilidad psicosocial o económica (Instituto Colombiano de Medicina Legal y Ciencias Forenses, 2015).

Es importante destacar que las estadísticas confirman estos postulados. El suicidio tiene comportamientos particulares de acuerdo con el nivel económico de los países e incluso esta característica influencia el método de 
ejecución del suicidio (OMS, 2012), de modo que resultan trascendentes las condiciones del contexto como determinantes sociales, económicos y culturales para los riesgos en salud.

El análisis de las condiciones económicas en los países con mayores tasas de suicidio indica que existe algún tipo de vulnerabilidad económica estable o transitoria, manifestada en el producto interno bruto bajo, una alta proporción de personas en pobreza multidimensional o crisis políticas-económicas-sociales que afectan la calidad de vida de la población, y que pueden progresar hacia alteraciones de la salud mental (ver tabla 1).

Tabla 1

Indicadores económicos en los diez países con mayor tasa de suicidio 2015

\begin{tabular}{|c|c|c|c|c|c|}
\hline $\begin{array}{l}\text { Ranking } \\
\text { países } \\
\text { suicidio }\end{array}$ & $\begin{array}{l}\text { Tasa } \\
\text { suicidio } \\
\text { países }\end{array}$ & $\begin{array}{c}\text { PIB } \\
2016\end{array}$ & $\begin{array}{c}\text { Pobreza } \\
\text { multi- } \\
\text { dimensional }\end{array}$ & Inflación & $\begin{array}{c}\text { Crisis } \\
\text { política, económica, social }\end{array}$ \\
\hline Guyana & 44,2 & $3,2 \%$ & $7 \%$ & No registra & Económica 2015 \\
\hline \multicolumn{6}{|l|}{ China } \\
\hline Corea del Norte & 38,5 & $0,8 \%$ & No registra & No registra & Económica 2010 Conflictos políticos 2015 \\
\hline Corea del Sur & 28,9 & $2,7 \%$ & No registra & $0,7 \%$ & Conflictos políticos 2015 \\
\hline Sri-Lanka & 28,8 & $4,8 \%$ & No registra & $0,9 \%$ & Antecedente de 26 años de Guerra Civil \\
\hline Lituania & 28,2 & $2,3 \%$ & $22,2 \%$ & $-0,9 \%$ & No crisis económica - Incremento de riesgo de pobreza \\
\hline Surinam & 27.8 & $-0,3 \%$ & $25,9 \%$ & $6,9 \%$ & Económica 2013-2016 \\
\hline Mozambique & 27.4 & $6,6 \%$ & $70.2 \%$ & $3,6 \%$ & Conflicto político-militar, Económica \\
\hline Tanzania & 24.9 & $7 \%$ & $66.4 \%$ & $5,6 \%$ & Crisis humanitaria Migrantes 2015 \\
\hline Nepal & 24.9 & $2,7 \%$ & $26.6 \%$ & $7,9 \%$ & Crisis humanitaria Terremoto-Crisis Económica 2015 \\
\hline Kazajstán & 23.8 & $1,2 \%$ & $1,1 \%$ & No registra & Afectado por conflictos de países vecinos 2014 \\
\hline Burundi & 23.1 & $-4,0 \%$ & $81,8 \%$ & $5,6 \%$ & Crisis política 2015 \\
\hline
\end{tabular}

Nota. Elaborado a partir de datos del Banco Mundial (s. f.).

En el 2015, Santa Marta (Magdalena), ciudad ubicada al norte de Colombia y la segunda más antigua de Suramérica, la tasa de suicidio consumado fue de 5,58 (24) casos por cada 100000 habitantes. Esta cifra la situaba entre las once ciudades de mayor ocurrencia junto con Bogotá, Villavicencio, Pasto, Cúcuta, Pereira, Bucaramanga, Ibagué, Medellín, Barranquilla y Cartagena (Instituto Colombiano de Medicina Legal y Ciencias Forenses, 2015).

El análisis de las condiciones económicas de las ciudades en Colombia con mayores tasas de suicidio para el 2015 muestra en estos contextos algún tipo de vulnerabilidad manifestada en indicadores económicos como pobreza, desempleo, necesidades básicas insatisfechas y desigualdad. En este punto, es necesario resaltar el comportamiento de los datos: no todas las ciudades presentan todos los indicadores económicos afectados. Parece ser que se compensan en su existencia, de modo que todas las ciudades del ranking presentan al menos una característica económica desfavorable. Por ejemplo, Bogotá como primera ciudad con mayores casos de suicidio no registra porcentaje representativo de población en pobreza, en comparación con ciudades como Bucaramanga o Santa Marta, pero sí registra un Gini de desigualdad superior al de todas las ciudades del ranking. Para el caso de Pasto, se destaca la pobreza y el desempleo; para Cartagena, la pobreza y necesidades insatisfechas; y en el caso de Cúcuta, el desempleo y las necesidades insatisfechas (ver tabla 2). 
Tabla 2

Indicadores económicos en las once ciudades de Colombia con mayor tasa de suicidio 2015

\begin{tabular}{|c|c|c|c|c|c|c|}
\hline Ciudades Colombia & $\begin{array}{l}\text { Casos } \\
\text { suicidio }\end{array}$ & $\begin{array}{l}\text { Tasa suicidio x } \\
100000 \text { hab. }\end{array}$ & $\begin{array}{c}\text { Indicador económico } \\
\text { pobreza }\end{array}$ & $\begin{array}{l}\text { Indicador } \\
\text { económico } \\
\text { desempleo }\end{array}$ & $\begin{array}{c}\text { Indicador } \\
\text { Económico NBI }\end{array}$ & Gini \\
\hline Bogotá & 310 & 4,64 & $10,4 \%$ & $8,7 \%$ & $9,20 \%$ & 0,49 \\
\hline Medellín & 344 & 7,42 & $23,7 \%$ & $11,3 \%$ & $12,42 \%$ & 0,48 \\
\hline Pasto & 40 & 10,64 & $40 \%$ & $11 \%$ & $16,20 \%$ & 0,48 \\
\hline Barranquilla & 45 & 4,40 & $25,7 \%$ & $6,9 \%$ & $17,72 \%$ & 0,43 \\
\hline Ibagué & 33 & ?,11 & No registra & $13,3 \%$ & $16,23 \%$ & 0,44 \\
\hline Pereira & 28 & 6,98 & $22,3 \%$ & $13,6 \%$ & $13,37 \%$ & 0,43 \\
\hline Cartagena & 28 & 3,36 & $39,3 \%$ & $7,9 \%$ & $26,01 \%$ & 0,46 \\
\hline Villavicencio & 27 & 6,77 & $21,8 \%$ & $11 \%$ & $17,07 \%$ & 0,44 \\
\hline Cúcuta & 26 & 4,84 & No registra & $14,5 \%$ & $23,34 \%$ & 0,44 \\
\hline Bucaramanga & 23 & 5,05 & $40 \%$ & $9,4 \%$ & $11,55 \%$ & 0,40 \\
\hline Santa Marta & 24 & 3,86 & $44,8 \%$ & $10,9 \%$ & $29,03 \%$ & 0,45 \\
\hline
\end{tabular}

Nota. Elaborado a partir de datos del Instituto de Medicina Legal y Ciencias Forenses (2015) y del Departamento Administrativo Nacional de Estadística (DANE, 2011 y 2015).

El análisis particular de Santa Marta indica la presencia de diversos factores que pueden constituir riesgo en la población. Los informes gubernamentales de las condiciones socioeconómicas y sociales de Santa Marta (Colombia) no resultan tan favorables para ejercer un impacto positivo en el bienestar de sus habitantes. Para el 2011, el 23,5 \% de la población (282 557 personas) vivía en pobreza extrema con ingresos mensuales de 83578 pesos (estándar nacional de pobreza extrema para 2010). De acuerdo con el Boletín de Pobreza Monetaria y Multidimensional en Colombia, en el 2015 la ciudad de Santa Marta presentaba un índice de pobreza de 30,7, un índice de pobreza extrema de 6,5 y un Gini de 0,45 en desigualdad; estos resultados desfavorables la ubican entre diversas ciudades del país en el sexto lugar después de Quibdó, Riohacha, Cúcuta, Florencia y Popayán (DANE, 2015). Por otra parte, esta ciudad históricamente ha sido víctima de desplazamientos forzosos para un total 123652 personas violentadas entre 1999 y 2012; cifras que revelan un escenario afectado por el conflicto armado y potencialmente bajo diversas problemáticas sociales y de salud
(Consultoría para los Derechos Humanos y el Desplazamiento, 2012).

En el 2018, Santa Marta (Magdalena) presentaba una tasa de 4,56 (21) casos por cada 100000 habitantes. Esta cifra la situaba entre las diecisiete ciudades de mayor ocurrencia junto con Bogotá, Medellín, Cali, Barranquilla, Pasto, Manizales, Cartagena, Bucaramanga, Cúcuta, Pereira. Valledupar, Armenia, Ibagué, Popayán, Sincelejo y Villavicencio. El comportamiento de los datos para el 2018 muestra algunas variaciones respecto a la posición de las ciudades. La lista se organiza por número de casos y no de acuerdo con la tasa; por esta razón, el análisis debe tomarse con precaución. Particularmente en Santa Marta, el 14,3 \% de la población se encuentra con necesidades básicas insatisfechas, desempleo del 7,9\%, pobreza monetaria de 33,7 \% y un Gini de desigualdad de 0,46 . Al respecto, la evidencia señala asociación entre desigualdad económica y la tasa de intentos de suicidio, así como con la desigualdad del ingreso y el desempleo en población colombiana (ver tabla 3; Arenas et al., 2015; Dávila-Cervantes y Pardo-Montaño, 2017). 


\begin{tabular}{ccccccc}
\hline Ciudades Colombia & Casos suicidio & $\begin{array}{c}\text { Tasa suicidio } x \\
\text { 100 } 000 \text { hab. }\end{array}$ & $\begin{array}{c}\text { Indicador económico } \\
\text { pobreza }\end{array}$ & $\begin{array}{c}\text { Indicador } \\
\text { económico } \\
\text { desempleo }\end{array}$ & $\begin{array}{c}\text { Indicador } \\
\text { económico NBI }\end{array}$ & Gini \\
\hline Bogotá & 387 & 5,11 & 2,5 & $10,1 \%$ & $3,36 \%$ & 0,50 \\
Medellín & 172 & 7,21 & 2,7 & $10,3 \%$ & $5,19 \%$ & 0,47 \\
Cali & 119 & 5,25 & 3,5 & $10,6 \%$ & $4,08 \%$ & 0,46 \\
Barranquilla & 64 & 5,62 & 2,2 & $8,6 \%$ & $9,05 \%$ & 0,47 \\
Pasto & 44 & 10,35 & 4,2 & $7,6 \%$ & $8,11 \%$ & 0,44 \\
Manizales & 42 & 11,19 & 2,1 & $10,8 \%$ & $6,18 \%$ & 0,45 \\
Cartagena & 42 & 4,40 & 3,4 & $10,3 \%$ & $12,38 \%$ & 0,43 \\
Bucaramanga & 40 & 8,08 & 1,6 & $8,2 \%$ & $5,38 \%$ & 0,44 \\
Cúcuta & 39 & 6,37 & 7,0 & $15,7 \%$ & $16,69 \%$ & 0,44 \\
Pereira & 35 & 7,9 & 1,7 & $8,5 \%$ & $5,83 \%$ & 0,41 \\
Valledupar & 35 & 7,99 & 7,1 & $14,9 \%$ & $17,69 \%$ & 0,45 \\
\hline
\end{tabular}

Nota. Elaborado a partir de datos del Instituto de Medicina Legal y Ciencias Forenses (2019) y del DANE (2018a, 2018b, 2018c).

En general, las diferentes teorías sobre el suicidio en la adolescencia abordan el problema desde una perspectiva multifactorial. Se incluyen las condiciones socioeconómicas contextuales como factores de riesgo, y especialmente en la adolescencia, las características evolutivas como el aumento de demandas, inmadurez, soledad, poca tolerancia a la frustración, disfunción de la familia, dificultades académicas y desarraigo cultural; adicionalmente, el inicio de propios códigos de conducta, desarrollo del pensamiento formal, modificación del rol, búsqueda de la identidad sexual y personalidad, entre otros cambios psicológicos y biológicos (Cortés, 2014).

Aunque las circunstancias sociales, psicológicas y biológicas generan mayor vulnerabilidad, la explicación al por qué no todos los individuos bajo estas condiciones se precipitarían al suicidio le corresponde al campo de la psicología (Durkheim, 1987). Desde esta disciplina, aún son necesarios más estudios que se aproximen a la naturaleza de los comportamientos suicidas para la prevención efectiva. Dada esta necesidad, es importante reconocer los factores asociados desde una perspectiva de recursos psicológicos. Esta reflexión señalará que la regulación emocional es necesaria para la expresión, modulación y flexibilidad del autocontrol emocional (Sroufe, 2000), teniendo en cuenta que, en su opuesto, la desregulación se encuentra inmersa en distintas psicopatologías como trastornos de ansiedad, trastornos depresivos y trastornos de personalidad (Hervás, 2011).

\subsection{VISLUMBRAR LA REGULACIÓN EMOCIONAL DESDE GROSS}

Para comprender la regulación emocional, debemos reconocer que la experiencia de una emoción es distintiva a los mecanismos para la regulación de emociones positivas y negativas. Las emociones son respuestas esenciales para la supervivencia al propiciar la adaptación a través de las relaciones sociales, la adquisición del aprendizaje y la activación de la huida ante el peligro (Gross, 1999). Las emociones acompañan el despliegue cognitivo y dirigen el comportamiento humano; no obstante, la gestión emocional puede generar 
efectos negativos o positivos para la salud mental. En este sentido, la adecuada gestión puede facilitar la toma de decisiones y la resolución de problemas en las negociaciones interpersonales; o contrariamente, la gestión inadecuada derivada del ambiente, intensidad o temporalidad de la emoción puede ser problemática para la convivencia (Werner y Gross, 2010).

Precisamente, la regulación emocional se centra en el mantenimiento, mejora o minimización de las respuestas emocionales en contexto; la gestión de estas respuestas puede ser de forma extrínseca, como el contexto orientador de la regulación, o intrínseca, como los propios esfuerzos cognitivos-conductuales que realizan las personas (Bridges et al., 2004; Campos et al., 2004; Cole et al., 2004; Gross y Thompson, 2007; Thompson, 1994). En este punto, debemos considerar que la interacción de los mecanismos intrínsecos y extrínsecos es necesaria en la regulación emocional exitosa.

El mantenimiento, mejora o minimización de las respuestas emocionales exige monitoreo y evaluación para la modificación de la intensidad, temporalidad, forma y frecuencia de la experiencia emocional (Thompson, 1994). En otras palabras, el metaconocimiento emocional es fundamental en los procesos de regulación de emociones. En la denominada experiencia emocional, intervienen componentes como respuesta fisiológica, expresión verbal, expresión no verbal y conducta, que la posicionan como una experiencia subjetiva e irrepetible entre individuos aún en la misma situación y frente a la misma emoción.

Para vislumbrar la regulación emocional, debemos acercarnos a la generación de una emoción desde la presencia de un evento o situación, la atención sobre el evento o situación, la evaluación del evento o situación, la respuesta emocional ante el evento o situación y la maleabilidad o manejo de la respuesta emocional (Lazarus, 1966; Gross, 2008).
Independientemente de la perspectiva teórica de la emoción, estas características en general se encuentran presentes (Barrett et al., 2006; Barrett et al., 2007; Ekman et al., 1972; Frijda, 1986; Levenson, 1994; Gross y Thompson, 2007).

De forma específica, nos acercaremos al modelo modal de la emoción, que traza el procesamiento de una emoción desde la situación, la atención, la evaluación y la respuesta emocional. Las respuestas emocionales derivadas corresponden a emociones básicas y a múltiples combinaciones de emociones que se expresan de forma particular en determinada situación. Estas respuestas modifican la situación inicial a partir de la evaluación, por tanto, no es un proceso constantemente lineal (Ortony y Turner, 1990).

La regulación emocional se inserta en el procesamiento de la emoción en las denominadas estrategias de regulación emocional. Las estrategias de regulación pueden ser diversas de acuerdo con la perspectiva teórica, y en general se clasifican según el lugar del procesamiento donde se introducen: antecedente y respuesta emocional. La regulación emocional insertada en los antecedentes se efectúa así: 1) seleccionar la situación, permite ejecutar una acción para participar o no de un evento que puede ser fuente de emociones que necesitan ser reguladas; 2) modificar la situación, en algunas ocasiones es posible cambiar la situación para reducir el impacto de la respuesta emocional; 3) atender, podemos direccionar la atención en un detalle de la situación para impactar la respuesta emocional; 4) cambiar la evaluación cognitiva, consiste en modificar la evaluación otorgando un nuevo significado. Mientras, la regulación emocional insertada en la respuesta puede efectuarse así: 5) modular la respuesta, se suprime la expresión de la respuesta fisiológica o comportamental (Gross, 1999).

Las anteriores conforman dos estrategias de regulación emocional denominadas supresión 
y revaluación cognitiva. La primera está centrada en la respuesta emocional, es referente a inhibir la expresión de una emoción a nivel afectivo, fisiológico, facial, verbal, gestual, entre otros recursos cognitivos e interpersonales (Gross y Levenson, 1993; Gross, 1998a y 1998b). Mientras que la segunda consiste en transformar la interpretación de la situación (Gross y John, 2003). En esta estrategia, el lenguaje es el canal para construir y reconstruir significados a través de la interacción inter e intrapersonal. Finalmente, las emociones son categorías constructivistas e intersubjetivas con componentes semánticos (Goldin et al., 2008).

\subsection{LA REGULACIÓN EMOCIONAL EN CONTEXTOS DE DESVENTAJA SOCIAL Y ECONÓMICA}

La dimensión emocional tiene vital importancia en la adolescencia cuando es notoria la expresión afectiva en relación con amar a otros, amarse a sí mismo, pero sobre todo cuando los procesos de autorregulación continúan en desarrollo, y se manifiesta impulsividad y diferentes conductas de riesgo (Oliva, 2012 y 2014). Precisamente, las estrategias inadecuadas y las dificultades en habilidades emocionales obstaculizan los esfuerzos por la regulación exitosa. Cuando las emociones negativas como la tristeza, la ira o el miedo se manifiestan de manera excedida y se evidencia una falla en la autogestión, se afecta el bienestar social y psicológico (Hervás, 2011). Por el contrario, la regulación emocional exitosa permitiría al individuo el control del monitoreo, evaluación y modificación de la intensidad y duración de sus emociones por medio de procesos extrínsecos e intrínsecos (Thompson, 1994).

No obstante, llevar a cabo una regulación exitosa no es sencillo. Existen diversos factores económicos, sociales y hasta culturales que afectan el despliegue de la regulación: ingreso económico, estructura familiar, inequidad social, acceso a los servicios de salud, acceso a la educación, violencia, nutrición, poco soporte social, y clima social y político, entre otros (Morris et al., 2007). El adolescente se encuentra en una transición caracterizada por la regulación parental, impuesta por madre-padre, y progresa a controlar sus propios procesos emocionales (Aldrete-Cortez et al., 2014). Ante esta trasformación debe sobrellevar los factores sociales y económicos que obstaculizan el despliegue de la autorregulación.

Desde el modelo de Gross, las estrategias de regulación como supresión de la expresión y revaluación cognitiva pueden generar efectos negativos o positivos. Por ejemplo, la revaluación cognitiva permite afrontar emociones negativas ante estímulos aversivos; en consecuencia, impacta el despliegue fisiológico (como ritmo cardíaco y conductancia de la piel). Debe tenerse en cuenta que la revaluación cognitiva requiere de entrenamiento en recursos cognitivos. Por su parte, la estrategia de supresión actúa sobre la experiencia afectiva al disminuir estados internos. La supresión también implica un gran esfuerzo para el individuo al requerir automonitoreo y autocorrección. Esta estrategia se manifiesta en la anulación de la expresividad emocional e incrementa el afecto negativo, por tanto, no es favorable en la adversidad.

Finalmente, debe ser un imperativo el reconocimiento del contexto social y económico para comprender el acceso a las estrategias de regulación emocional. Los adolescentes en desventaja deben enfrentar la hostilidad del medio, y en consecuencia acceden a estrategias de regulación rápidas y adaptativas, pero en el momento inmediato (Thompson y Calkins, 1996; Thompson et al., 1995).

\subsection{OTRAS ESTRATEGIAS PARA LA GESTIÓN DE EMOCIONES}

El modelo de regulación emocional de Gross (2007) agrupa las estrategias de afrontamiento posibles durante el proceso de regulación, 
adoptando diferentes modelos (Skinner et al., 2003; Augustine y Hemenover, 2008; Larsen y Prizmic, 2004; Páez et al., 2012). Algunas de las estrategias tienen efectos negativos para la salud; no obstante, la subjetividad de la experiencia emocional sugiere que dependerá de la emoción, activación fisiológica, historia del sujeto, patrones culturales, etc.

\section{Selección y modificación de la situación}

$\checkmark$ Resolución de problemas o planificación

$\checkmark$ Indefensión aprendida*

$\checkmark$ Abandono psicológico*

$\checkmark$ Apoyo social instrumental o informativo

$\checkmark$ Búsqueda de información

$\checkmark$ Altruismo

$\checkmark$ Aislamiento*

\section{Atención e interpretación}

$\checkmark$ Reevaluación positiva

$\checkmark$ Distracción

$\checkmark$ Rumiación*

$\checkmark$ Evitación*

$\checkmark$ Comparación social

\section{Modificación de la respuesta emocional}

$\checkmark$ Regulación fisiológica

$\checkmark$ Relajación

$\checkmark$ Aceptación

$\checkmark$ Gratitud

$\checkmark$ Minimización del problema

$\checkmark$ Autorreconfortarse

$\checkmark$ Sentido del humor

$\checkmark$ Inhibición emocional*

$\checkmark$ Escribir / expresión regulada

\section{Cambio de la expresión emocional}
$\checkmark$ Catarsis*
$\checkmark$ Confrontación 


\subsection{LA REGULACIÓN EMOCIONAL Y LA CONDUCTA SUICIDA}

Algunos estudios registran el vínculo entre la regulación emocional y el suicidio, posicionando a la supresión emocional como una estrategia de frecuente uso en diferentes comportamientos suicidas (Victor y Klonsky, 2014; Shelef et al., 2015; Adrian et al., 2011). Generalmente, esta interacción es evidente por las emociones asociadas a la conducta suicida y, de forma específica, las emociones negativas intensas previas a los intentos de suicidio con y sin fines de muerte. De acuerdo con el registro, las autolesiones se ejecutan por la búsqueda de alivio del afecto negativo, autocastigo, antidisociación, influencia interpersonal, búsqueda de sensaciones, entre otros (Klonsky, 2007). Así mismo, se asocian a la insatisfacción con la vida (Victor y Klonsky, 2014), la desesperación abrumadora, la vergüenza, el odio a sí mismo, la alienación y el aislamiento personal (Everall et al., 2006), y el dolor emocional (Levi et al., 2011; Shelef et al., 2015).

En contextos de desventaja social y económica, la supresión de la expresión está presente en adolescentes con riesgo suicida (Suárez, 2017). Precisamente, esta interacción de factores posiciona la complejidad de la regulación emocional ante la vulnerabilidad (Thompson y Goodman, 2010). La supresión de las emociones podría ser un multiplicador del riesgo suicida, porque se interpone ante la búsqueda de ayuda o de apoyo con padres-pares u otros. No obstante, es necesario considerar las necesidades individuales en regulación emocional, es decir, cada individuo en contextos de riesgo tiene sus propias necesidades de regulación y determinadas estrategias pueden ser funcionalmente adaptativas, mientras otras no. Por ejemplo, es posible que si las ideas suicidas persisten luego de un intento o en ausencia de un intento y estas son rígidas, aceptadas y seguras con una planificación detallada y sin contemplación de factores disuasorios, la expresión emocional será necesaria pero no suficiente porque se necesitará revaluar cognitivamente; mientras que ante el carácter de ambivalencia ante la idea de suicidio, la expresión emocional podría funcionar porque permite la búsqueda de ayuda-proximidad para obtener una nueva perspectiva u otras estrategias que permitan recuperar el bienestar.

Antes, se ha expuesto el modelo de Gross de regulación emocional con especial mención de las estrategias de supresión emocional y revaluación cognitiva, aunque otros estudios señalan la no aceptación y poca autoconciencia asociadas a los comportamientos suicidas (Pisani et al., 2013; Rajappa et al., 2012; Ghorbani et al., 2017). Es necesario reconocer que las características de regulación emocional pueden ser moderadas por la presencia de psicopatología (Berzenski, 2019). Otras estrategias como conciencia emocional, claridad emocional, modulación flexible, autocontrol, aceptación e incluso la facilidad para acceder a estrategias de regulación pueden tener un rol fundamental, aunque no existe claridad de su contribución (Wolff et al., 2019). Se recomiendan otras estrategias consideradas adaptativas como la revaluación, la distracción y la atención plena.

Determinados comportamientos suicidas funcionan como estrategias de afrontamiento negativas evitativas o distractoras de la experiencia emocional, regulan la experiencia física y emocional (Nock y Prinstein, 2004), y también producen sensación de alivio del dolor emocional a través del dolor físico (Wolf et al., 2019). No obstante, aún no son suficientemente claras las necesidades específicas de regulación emocional ante la conducta suicida y los perfiles de riesgo fundamentados en las necesidades de regulación (Peterson et al., 2019).

Diferentes modelos de regulación emocional intentan aproximarse a comprender el suicidio, a través de diversas perspectivas que pueden nublar el panorama de la regulación 
emocional y la conducta suicida, si no se intenta considerar un modelo integrador. Por el momento, es claro que las teorías sobre el suicidio coinciden en la existencia de mecanismos distintivos entre individuos con y sin tendencias suicidas (Albanese et al., 2019).

\subsection{IDENTIFICACIÓN DE LAS NECESIDADES PSICOAFECTIVAS DE LOS ADOLESCENTES}

En la actualidad, las necesidades psicoafectivas de los adolescentes son diversas y también dependientes de la cultura, la historia familiar, condiciones socioeconómicas, eventos estresantes vitales, exposición a la violencia y otras experiencias adversas. Al utilizar un ejercicio de expresión de emociones en aproximadamente 1141 adolescentes, se identificaron algunas necesidades psicoafectivas.

Los adolescentes necesitan establecer relaciones de confianza-comunicación con padres basadas en la expresión emocional que fortalezca el vínculo afectivo. La confianza-comunicación debe ser fuente de apoyo socioemocional y vía para fortalecer las creencias de eficacia de los adolescentes (Kogut, 2016), así como medio para estimular la restructuración de pensamientos irracionales. Aún más, cuando la confianza-comunicación se convierte en un sistema de autorregulación emocional y conductual, es decir, el vínculo fundamentado en estas características puede facilitar el uso-acceso de estrategias de regulación emocional por el individuo (Couto y Tavares, 2016; Muñoz, 2017).

"Me siento muy cansado debido a la falta de confianza de mi familia hacia mí, aunque en ocasiones digan que me apoyan, yo siento que me creen incapaz de lograr las cosas...".

\section{Adolescente 1}

"Me siento un poco aburrida, deprimida porque pensaba que las cosas iban a cambiar con mi familia, pero siguen iguales; mi mamá todo el tiempo con una actitud grosera todavía por lo que pasó... Me gustaría estar bien con ella otra vez, y recuperar esa confianza, no es fácil, pero si ella me escucha, sería mejor y pues no me sintiera como me estoy sintiendo...".

\section{Adolescente 6}

"La relación con mi padre está rota. Siempre hice todo lo posible por hacerlo sentir orgulloso, pero nunca ha sido suficiente... Siempre celebró mis aciertos, pero nunca me dio su mano cuando fallé... Los abrazos y palabras de afecto siempre las propuse yo".

\section{Adolescente 4}

Las figuras de cuidado son fundamentales en el desarrollo humano; la presencia de un individuo que represente protección, afecto y atención disminuye el riesgo de victimizaciones que afectan los recursos psicológicos, en el uso de estrategias de regulación emocional poco efectivas. El soporte que requieren los adolescentes está relacionado con una estructura que proporcione, además, autoconfianza y autoeficacia. Las dificultades de regulación emocional y la falta de adultos de confianza se asocian a mayor riesgo de suicidio, más allá de los efectos de los síntomas depresivos y factores demográficos (Pisani et al., 2013).

"Crecí sin mis padres... Sufrí mucha discriminación y me sentí señalado por todos... Soy una persona muy orgullosa y creo que esto se debe a crecer $\sin$ mucho cariño, afecto...".

\section{Adolescente 2}

"El no conocer a la persona que tenía que haber llamado padre me ha marcado... En ocasiones eso me hace sentir menos que los demás...".

\section{Adolescente 3}

La gestión emocional en la adolescencia no es sencilla. Los adolescentes deben aprender a 
afrontar las emociones negativas y positivas, a pesar de las condiciones del contexto y la educación emocional recibida (Lemaigre y Taylor, 2019).

"Quiero expresar el amor que le tengo a mis padres, aunque no se los demuestre porque ellos tampoco es que me lo demuestren con hechos hacia mí, de pronto por eso no lo hago".

\section{Adolescente 5}

De forma especial, es un reto la educación emocional para el afrontamiento del dolor producto de las experiencias adversas en la infancia y la adolescencia. Los adolescentes con riesgo suicida informan una experiencia emocional negativa, intensa e intolerable, asociado a la inadecuación, no pertenencia y sobrecarga (Joiner et al., 2005; Van Orden et al., 2010).

"Generalmente yo me siento aburrida con $\mathrm{mi}$ vida por sentir que es una rutina, siento siempre dolor y trato de mostrar felicidad hacia los que me rodean. Me siento estresada por mi vida y por los que están a mi alrededor. Creo que estoy deprimida y no sé cómo cambiar esa situación".

\section{Adolescente 7}

"Me siento muy triste, al hacer un recorrido en mi vida, igual llego a la conclusión de que todo me sale mal. Solo veo fracasos. Solo quiero irme lejos y estar tranquila sin sentirme fracasada ni frustrada por como soy".

\section{Adolescente 8}

"Me siento agotada, poco valorada, todos los esfuerzos son poco valorados, en vez de recalcar las cosas buenas que hago, se me juzga y reprocha".

\section{Adolescente 9}

"He tenido rabia por mucho tiempo, rabia de mí misma por no poder ser quien soy, por tener miedo siempre y estar nerviosa cada día... También he tenido rabia por mucho tiempo de mi papá, por dejarnos, porque no fuimos su prioridad... Y no quiero seguir teniendo ni rabia, ni la ira tan tremenda que siento, ni los nervios, ni las inseguridades, ni resentimientos... Quiero poder sentir amor, solo eso".

\section{Adolescente 11}

La supresión de la expresión como estrategia de regulación emocional centrada en la respuesta emocional es la estrategia de mayor acceso ante eventos que no pueden modificar los adolescentes (Suárez, 2017).

"Después de estar años en el clóset, uno aprende a suprimir sus emociones tan bien que después resulta difícil reaprender a expresarlas".

\section{Adolescente 10}

\section{EPílogo}

Al analizar la regulación emocional en la adolescencia, son evidentes las dificultades para responder ante la experiencia de emociones negativas intensas, de modo que el adolescente necesita recursos para hacer frente a sus emociones. Además, los procesos emocionales desregulados en adolescentes se afianzan ante los conflictos familiares y el poco apoyo en la gestión de emociones; por consiguiente, se incrementa el riesgo de autolesiones no letales (Adrian et al., 2011). Sin embargo, algunos cambios en el desarrollo del procesamiento cognitivo-emocional de los adolescentes pueden modificar este riesgo (Voon et al., 2014).

Es necesario recordar que la supresión de emociones como estrategia de regulación emocional es una estrategia relacionada con la psicopatología y conductas suicidas (Kring y Sloan, 2010; Najmi et al., 2007); sin embargo, los estudios no permiten generalizar esta condición (Voon et al., 2014). Aunque un menor uso de la 
revaluación cognitiva incrementa el riesgo de autolesiones durante un año (Voon et al., 2014).

En el despliegue del presente documento, se referenció la regulación emocional como una de las estrategias que se introducen en el procesamiento de una emoción para el mantenimiento, mejora o minimización de la respuesta emocional en contexto, con el fin de señalar que si la experiencia emocional es subjetiva, las estrategias de regulación emocional no deben ser generales para los grupos, sino particulares para los individuos, con características diferenciales; más aún, cuando las desventajas sociales y económicas configuran factores para el riesgo de suicidio.

Respecto al riesgo suicida en adolescentes, es necesario reconocer que dadas las características evolutivas y las deficiencias en recursos personales relacionadas con la poca tolerancia a afrontar el estrés de la vida, los comportamientos suicidas pueden ser explicados por una reacción a una situación estresante de carácter temporal (Pérez, 2010) y el individuo puede o no recuperar su salud psicológica (Schotte y Clum, 1987), dependiendo de la persistencia de factores como la depresión y el historial de intentos (Apter et al., 1988).

Realmente, son múltiples las posibles combinaciones del riesgo suicida, lo que configura un fenómeno complejo de explicar y predecir en los adolescentes, sobre todo por las características evolutivas que construyen un sistema de vulnerabilidad junto con las fallas del contexto social, económico, político, y psicológiCo (Mack, 1986). El modelo arquitectónico para el suicidio explica que factores de riesgo del macrocosmos como las condiciones sociales y económicas, y factores biopsicológicos como la vulnerabilidad biológica, experiencias del desarrollo, personalidad-autoestima, relaciones con padres-pares, psicopatología, ontogenia y situaciones estresantes, propician las conductas autoflingidas (Mack, 1986).
Como se mencionó, las desventajas sociales y económicas incrementan la vulnerabilidad y la desintegración/exclusión social y, en consecuencia, obstaculizan la supervivencia cuando estas interfieren en la salud o impiden la realización de metas personales cuando no existen oportunidades para el acceso o avance en la educación; además, pueden producir desintegración, desadaptación, poca participación y bajo apoyo social.

Es claro que las circunstancias psicosociales adversas relacionadas directamente con la pobreza (como falta de alimentos, falta de acceso a servicios públicos, dificultades de acceso a la educación, falta de oportunidades para la profesionalización, la precariedad de la vivienda, falta de apoyo social, hechos victimizantes en la escuela, comunidad, o propios del sistema político-social) obstaculizan el desarrollo humano, cuando conducen a los adolescentes al trabajo a temprana edad, a la explotación sexual comercial, a la vinculación con bandas criminales o pandillas, al consumo de sustancias activas, entre otros. Al mismo tiempo, este factor puede moldear la estructura personal del adolescente, y puede en algunos casos ser determinante en el despliegue de recursos psicológicos.

Las desventajas sociales y económicas afectan el bienestar psicosocial en la infancia-adolescencia; por tanto, es indispensable orientar las políticas públicas a garantizar el derecho a la vida, la integridad física, la salud, la seguridad social, la alimentación equilibrada, su nombre-nacionalidad, a tener una familia y no ser separados de ella, al cuidado-amor, la educación, la cultura, recreación y a la libre expresión de su opinión, evitando ante todo el abandono, violencia física o moral, secuestro, venta, abuso sexual, explotación laboral o económica y trabajos riesgosos; en sí, el cumplimiento de los derechos de los niños y adolescentes (República de Colombia, 1991). Las experiencias adversas infantiles son 
importantes factores de riesgo para la salud en la adultez (Norman et al., 2012), y se agravan con la privación socioeconómica, redes sociales reducidas, problemas de salud mental y dificultad para buscar ayuda.

La desventaja social-económica incrementa progresivamente el bajo apoyo y aislamiento social (Fergusson et al., 2000), importantes factores de riesgo para la conducta suicida, al mismo tiempo que afecta los factores protectores. En líneas anteriores, se mencionó cómo la desintegración social desde la posición sociológica podría explicar el suicidio; desde esta perspectiva inhibirse socialmente disminuye las fuentes de apoyo social, a su vez que se ajusta a la percepción de sobrecarga y sentimientos de no pertenencia en individuos con tendencias suicidas (Joiner et al., 2005; Van Orden et al., 2010). Claramente, el aislamiento social puede configurarse por rasgos de personalidad, psicopatologías severas, además de intensas experiencias adversas infantiles (Masumoto et al., 2016; Lemaigre y Taylor, 2019) Así mismo, los individuos con esta tendencia pueden mostrar mayor preferencia por estrategias evitativas como la supresión de la expresión emocional (Zhao et al., 2014).

Las estrategias de regulación emocional centradas en la modulación de la respuesta emocional indican que la supresión de la expresión es una estrategia que afecta la salud psicológica. Por el contrario, la expresión emocional adecuada es necesaria en la supervivencia, y es necesario adquirir esta habilidad desde el aprendizaje cultural y familiar para la prevención del suicidio.

Aunque la gestión de emociones es un proceso que se especializa durante el ciclo vital, los actuales retos para la prevención conducen a la psicología a inclinarse a fortalecer los recursos personales por medio de la educación emocional. Cuando los seres humanos no logran activar la resistencia ante la adversidad, cuando fallan los procesos innatos-biológicos y la especialización de funciones durante el desarrollo se trunca, se observarán serias dificultades de los adolescentes para gestionar las emociones; la educación emocional podría restaurar la conciencia y autogestión emocionales (Bisquerra, 2003).

La alfabetización emocional podría configurar un capital de recursos psicológicos necesarios para padres e hijos permitiendo: a) mayor empatía ante las necesidades de cada etapa del desarrollo; b) comprender, aceptar y respetar los significados de vida en el desarrollo; c) modular la respuesta emocional o el proceso que interviene en la respuesta emocional negativa y dañina; d) dirigir la experiencia emocional a favor del bienestar; e) fortalecer las relaciones con los miembros próximos; f) fortalecer relaciones con miembros de la red social; y g) generar vínculos positivos con los miembros que se encuentran fuera de la red social. Estos recursos psicológicos que se adquieren de la educación emocional son una alternativa para el desarrollo humano, la salud y la calidad de vida; además, son una vía para la prevención del suicidio (Suárez, 2010, 2011 y 2012).

El suicidio en adolescentes es difícil de predecir cuando los factores de riesgo interactúan en diferentes combinaciones y aún no se registran combinaciones operacionales (Campo-Arias y Suárez-Colorado, 2019), porque pueden ser innumerables frente a las complejas problemáticas de la realidad, por ejemplo, el bullying, la desintegración familiar o la exposición a determinados juegos tecnológicos de riesgo (estos últimos conducen sistemáticamente al suicidio a través de una exposición progresiva a la idea de la muerte). La exposición a la violencia o experiencias adversas propician una regulación emocional disfuncional, y la tendencia suicida en entornos empobrecidos (Lemaigre y Taylor, 2019).

La prevención del suicidio en adolescentes debe considerar los factores de riesgo que 
progresivamente generan tolerancia a las ideas de la muerte (Joiner, 2005; Teismann et al., 2014). Es claro que la exposición metódica de factores de riesgo en corto tiempo estimula la generación de emociones negativas intensas, lo que incrementaría el nivel de estrés en la vida o el estado sistémico de alta vulnerabilidad por medio de autolesiones no letales, problemas del sueño, exposición a estímulos aversivos, introyección del significado e identificación con la figura de la muerte, estrés producto del riesgo por la muerte, soledad, legitimización entre pares por la experiencia compartida, incertidumbre, y finalmente la autolesión letal (Carli et al., 2014).

Por otra parte, las estrategias de prevención del suicidio basadas en el fomento de recursos psicológicos como la regulación emocional deben adecuarse a las condiciones sociales y económicas del contexto. Si la regulación emocional es un esfuerzo intrínseco, los adolescentes vulnerables requieren ser educados y reeducados a partir de los recursos disponibles; si la regulación emocional tiene mecanismos extrínsecos, los adolescentes requieren ser educados para las situaciones que no pueden modificar. Los proyectos que educan para la regulación emocional deben situarse en el contexto de desventaja social y económica. Así también, en el diseño de proyectos se debe reconocer la temporalidad de implementación que se requiere para educar en la revaluación cognitiva en grupos, donde la educación no fomenta el autoconocimiento/metaconocimiento emocional. Algunos estudios sugieren que las intervenciones psicosociales basadas en la revaluación cognitiva pueden ser efectivas teniendo en cuenta que los pacientes hospitalizados por intentos de suicidio requieren de estrategias adaptativas para gestionar las emociones negativas que buscaron reducir a través de la autolesión (Kiosses et al., 2018).

Finalmente, queremos insistir en la educación para gestionar adecuadamente las emociones con la finalidad de afrontar la competitividad y la supervivencia. No podemos evitar la adversidad, pero podemos aprender a responder desde las estrategias de regulación emocional más funcionales para prevenir el suicidio. Comprender la funcionalidad de las estrategias de regulación emocional en los diferentes comportamientos suicidas es un reto, de modo que se recomiendan más investigaciones referentes a las particularidades de la regulación emocional en contextos vulnerables.

Nota: Este texto no manifiesta la posición del autor sobre ningún modelo económico y es una aproximación parcial a un problema multifactorial. 


\section{REFERENCIAS}

Adrian, M., Zeman, J., Erdley, C., Lisa, L. y Sim, L. (2011). Desregulación emocional y dificultades interpersonales como factores de riesgo de autolesión no suicida en adolescentes. Revista de Psicología Infantil Anormal, 39(3), 389-400.

Aldrete-Cortez, V., Carrillo-Mora, P., Mansilla-Olivares, A., Schnaas, L. y Esquivel-Ancona, F. (2014). From Emotional and Cognitive Regulation to Self-Regulation Development in the First Year of Life. Anuario de Psicología/The uB Journal of Psychology, 44(2), 199-212.

Albanese, B. J., Macatee, R. J., Stanley, I. H., Bauer, B. W., Capron, D. W., Bernat, E., Joiner, T. y Schmidt, N. B. (2019). Differentiating Suicide Attempts and Suicidal Ideation using Neural Markers of Emotion Regulation. Journal of Affective Disorders, 257(1), 536-550.

Arenas, A., Gómez-Restrepo, C. y Rondón, M. (2016). Factores asociados a la conducta suicida en Colombia. Resultados de la Encuesta Nacional de Salud Mental 2015. Revista Colombiana de Psiquiatría, 45(1), 68-75.

Apter, A., Bleich, A., Plutchik, R., Mendelsohn, S. y Tyano, S. (1988). Suicidal Behavior, Depression, and Conduct Disorder in Hospitalized Adolescents. Journal of the American Academy of Child \& Adolescent Psychiatry, 27(6), 696-699.

Aron, R. (1980). Las etapas del pensamiento sociológico. Ediciones Siglo Veinte.

Augustine, A. A. y Hemenover, S. H. (2008). On the Relative Effectiveness of Affect Regulation Strategies: A Meta-Analysis. Cognition and Emotion, 23(6), 1181-1220.

Barrett, L. F., Mesquita, B., Ochsner, K. N. y Gross, J. J. (2007). The Experience of Emotion. Annual Review of Psychology, 58(1), 373-403.

Barrett, R. D. H., MacLean R. C. y Bell, G. (2006). Mutations of Intermediate Effect are Responsible for Adaptation in Evolving Pseudomonas Populations. Biology Letters, 2(2), 236-238.

Banco Mundial. (s. f.). Indicadores de desarrollo. https://datos.bancomundial.org/indicador

Berzenski, S. R. (2019). Distinct Emotion Regulation Skills explain Psychopathology and Problems in Social Relationships following Childhood Emotional Abuse and Neglect. Development and Psychopathology, 31(2), 483-496.

Bisquerra, R. (2003). Educación emocional y competencias básicas para la vida. Revista de Investigación Educativa, 21(1), 7-43.

Bridges, L. J., Denham, S. A. y Ganiban, J. M. (2004). Definitional Issues in Emotion Regulation Research. Child Development, 75(2), 340-345.

Campos, J. J., Frankel, C. B. y Camras, L. (2004). On the Nature of Emotion Regulation. Child Development, 75(2), 377-394.

Campo-Arias, A. y Suárez-Colorado, Y. (2019). ¿Es el suicidio un evento prevenible? Revista Salud UIS, 51(3), 187-199.

Carli, V., Hoven, C. W., Wasserman, C., Chiesa, F., Guffanti, G., Sarchiapone, M., Apter, A., Balazs, J., Brunner, R., Corcoran, P., Haring, C., Iosue, M., Kaess, M., Kahn, J. P., Keeley, H., Postuvan, V., Saiz, P., Varnik, A., Wasserman, D. y Cosman, D. (2014). Un nuevo grupo de adolescentes con un riesgo "invisible" de psicopatología y comportamiento suicida: hallazgos del estudio SEYLE. World Psychiatry, 13(1), 78-86.

Cole, P. M., Martin, S. E. y Dennis, T. A. (2004). Emotion Regulation as a Scientific Construct: Methodological Challenges and Directions for Child Development Research. Child Development, 75(2), 317-333.

Company, R., Oberst, U. y Fernández, F. S. (2012). Regulación emocional interpersonal de las emociones de ira y tristeza. Boletín de Psicología, 104(1), 7-36.

Comisión Económica para América Latina y el Caribe. (2009). Los derechos económicos, sociales y los derechos culturales: instrumentos y obligaciones de los Estados en relación a las personas de edad. http://www.cepal.org/sites/ default/files/events/files/djaspers_0.pdf 
Consejo Económico y Social. (1997). Informe 8/1996 sobre la pobreza y la exclusión social en España. CES.

Couto Dias, V. V. y Tavares Araújo, M. D. S. (2016). Apego e risco de suicídio em adolescentes: estudo de revisão. Revista da SPAGESP, 17(2), 120-136.

Consultoría para los Derechos Humanos y el Desplazamiento. (2012). Estadísticas históricas de desplazamiento. http://www.codhes.org/index.php?option=com_si\&type=1

Cortés, A. (2014). Conducta suicida: adolescencia y riesgo. Revista Cubana de Medicina General Integral, 30(1), 132-139.

Dávila-Cervantes, C. A. y Pardo-Montaño, A. M. (2017). Impacto de factores socioeconómicos en la mortalidad por suicidios en Colombia, 2000-2013. Revista Gerencia y Políticas de Salud, 16(33), 36-51.

Departamento Administrativo Nacional de Estadística. (2011). Pobreza monetaria y multidimensional en Colombia 2011. https://www.dane.gov.co/files/investigaciones/condiciones_vida/pobreza/boletin_pobreza_2011.pdf Departamento Administrativo Nacional de Estadística. (2015). Pobreza monetaria y multidimensional en Colombia 2015. https://www.dane.gov.co/index.php/estadisticas-por-tema/pobreza-y-condiciones-de-vida/pobreza-y-desigualdad/pobreza-monetaria-y-multidimensional-en-colombia-2015

Departamento Administrativo Nacional de Estadística. (2018a). Necesidades básicas insatisfechas (NBI). https:// www.dane.gov.co/index.php/estadisticas-por-tema/pobreza-y-condiciones-de-vida/necesidades-basicas-insatisfechas-nbi

Departamento Administrativo Nacional de Estadística. (2018b). Pobreza y desigualdad. https://www.dane.gov. co/index.php/estadisticas-por-tema/pobreza-y-condiciones-de-vida/pobreza-y-desigualdad

Departamento Administrativo Nacional de Estadística. (2018c). Mercado laboral. https://www.dane.gov.co/index.php/estadisticas-por-tema/mercado-laboral/empleo-y-desempleo

Durkheim, E. (1897). Le suicide: étude de sociologie. Alcan.

Durkheim, É. (1987). Las reglas del método sociológico. Premiá Editores.

Ekman, P., Friesen, W. V. y Ellsworth, P. (1972). Emotion in the Human Face: Guidelines for Research and an Integration of Findings. Pergamon.

Everall, R. D., Bostik, K. E. y Paulson, B. L. (2006). Being in the Safety Zone: Emotional Experiences of Suicidal Adolescents and Emerging Adults. Journal of Adolescent Research, 21(4), 370-392.

Frijda, N. H. (1986). The Emotions: Studies in Emotion and Social Interaction. Cambridge University Press.

Fergusson, D. M., Woodward, L. J. y Horwood, L. J. (2000). Risk Factors and Life Processes Associated with the Onset of Suicidal Behavior during Adolescence and Early Adulthood. Psychological Medicine, 30(1), 23-39.

Galtung, J. (1985). Twenty-Five Years of Peace Research: Ten Challenges and Some Responses. Journal of Peace Research, 22(2), 141-158.

Goldin, P. R., McRae, K., Ramel, W. y Gross, J. J. (2008). The Neural Bases of Emotion Regulation: Reappraisal and Suppression of Negative Emotion. Biological Psychiatry, 63(6), 577-586.

Gross, J. J. (1998a). Antecedent and Response-Focused Emotion Regulation: Divergent Consequences for Experience, Expression, and Physiology. Journal of Personality and Social Psychology, 74(1), 224-237.

Gross, J. J. (1998b). The Emerging Field of Emotion Regulation: An Integrative Review. Review of General Psychology, 2(3), 271.

Gross, J. J. (1999). Emotion Regulation: Past, Present, Future. Cognition \& Emotion, 13(5), 551-573.

Gross, J. J. y John, O. P. (2003). Individual Differences in Two Emotion Regulation Processes: Implications for Affect, Relationships, and Well-Being. Journal of Personality and Social Psychology, 85(2), 348-362.

Gross, J. J. y Levenson, R. W. (1993). Emotional Suppression: Physiology, Self-Report, and Expressive Behavior. Journal of Personality and Social Psychology, 64(6), 970-986.

Gross, J. J y Thompson, R. A. (2007). Regulación de la emoción: fundamentos conceptuales. En J. J. Gross (ed.), Manual de regulación de las emociones (pp. 3-24). La Prensa de Guilford.

Gross, J. (2008). Regulación emocional. Manual de Emociones, 3(3), 497-513. 
Ghorbani, F., Khosravani, V., Bastan, F. S. y Ardakani, R. J. (2017). The Alexithymia, Emotion Regulation, Emotion Regulation Difficulties, Positive and Negative Effects, and Suicidal Risk in Alcohol-Dependent Outpatients. Psychiatry Research, 252(1), 223-230.

Hervás, G. (2011). Psicopatología de la regulación emocional: el papel de los déficits emocionales en los trastornos clínicos. Psicología Conductual, 19(2), 347-372.

Instituto Colombiano de Medicina Legal y Ciencias Forenses. (2015). Forensis 2014. Datos para la vida. Forensis, 16(1). https://www.medicinalegal.gov.co/cifras-estadisticas/forensis

Instituto de Colombiano de Medicina Legal y Ciencias Forenses. (2019). Forensis 2018. Datos para la vida. Forensis, 19(1). https://www.medicinalegal.gov.co/documents/20143/386932/Forensis+2018.pdf/be4816a43da3-1ff0-2779-e7b5e3962d60

Joiner, T. (2005). Why People Die by Suicide. Harvard University Press.

Kiosses, D. N., Alexopoulos, G. S., Hajcak, G., Apfeldorf, W., Duberstein, P. R., Putrino, D. y Gross, J. J. (2018). Cognitive Reappraisal Intervention for Suicide Prevention (CRISP) for Middle-Aged and Older Adults Hospitalized for Suicidality. The American Journal of Geriatric Psychiatry, 26(4), 494-503.

Klonsky, E. D. (2007). Las funciones de la deliberada autolesión: una revisión de las pruebas de examen. La Psicología Clínica, 27(2), 226-239.

Kogut, E. (2016). Adult Attachment Styles, Self-Efficacy, and Causal Attributional Style for Achievement-Related Failures. Learning and Individual Differences, 50(1), 64-72.

Larsen, R. J. y Prizmić, Z. (2004). Affect Regulation. En R. Baumeister y K. Vohs (eds.), Handbook of Self-Regulation Research (pp. 40-60). Guilford.

Lazarus, R. S. (1966). Psychological Stress and the Coping Process. McGraw-Hill.

Levenson, R. W. (1994). Human Emotion: A Functional View. En P. Ekman y R. J. Davidson (eds.), The Nature of Emotion. Fundamental Questions (pp. 123-126). Sage.

Lemaigre, C. y Taylor, E. P. (2019). Mediators of Childhood Trauma and Suicidality in a Cohort of Socio-Economically deprived Scottish Men. Child Abuse \& Neglect, 88(1), 159-170.

Levi, Y., Weinberg, S. y Nadjar, R. (2011). Support Groups for Suicide Survivors: Self-Regulation and Social Support as Mediating the Effect of Recovery. European Psychiatry, 26(1), 1626.

Mack, J. (1986). Adolescent Suicide: An Architectural Model. En G. Klerman (ed.), Suicide and Depression among Adolescents and Young Adults (pp. 53-76). American Psychiatric Press.

Morris, A. S., Silk, J. S., Steinberg, L., Myers, S. S. y Robinson, L. R. (2007). The Role of the Family Context in the Development of Emotion Regulation. Social Development, 16(2), 361-388.

Masumoto, K., Taishi, N. y Shiozaki, M. (2016). Age and Gender Differences in Relationships among Emotion Regulation, Mood, and Mental Health. Gerontology and Geriatric Medicine, 2(1-8).

Muñoz, L. M. (2017). La autorregulación y su relación con el apego en la niñez. Revista Latinoamericana de Ciencias Sociales, Niñez y Juventud, 15(2), 807-821.

Najmi, S., Wegner, D. M. y Nock, M. K. (2007). Thought Suppression and Self-Injurious Thoughts and Behaviors. Behaviour Research and Therapy, 45(8), 1957-1965.

Norman, R. E., Byambaa, M., De, R., Butchart, A., Scott, J. y Vos, T. (2012). The Long-Term Health Consequences of Child Physical Abuse, Emotional Abuse, and Neglect: A Systematic Review and Meta-Analysis. PLoS Medicine, 9(11), e1001349.

Nock, M. K. y Prinstein, M. J. (2004). A Functional Approach to the Assessment of Self-Mutilative Behavior. Journal of Consulting and Clinical Psychology, 72(5), 885.

Nock, M. K., Prinstein, M. J. y Sterba, S. K. (2009). Revealing the Form and Function of Self-Injurious Thoughts and Behaviors: A Real-Time Ecological Assessment Study among Adolescents and Young Adults. Journal of Abnormal Psychology, 118(4), 816. 
Oficina del Alto Comisionado de las Naciones Unidas para los Derechos Humanos. (2009). Preguntas frecuentes sobre los Derechos Económicos, Sociales y Culturales. (Folleto Informativo 33). http://www.ohchr.org/Documents/Publications/FS33_sp.pdf

Oliva, A. (2012). Desarrollo cerebral y asunción de riesgos durante la adolescencia. Apuntes de Psicología, 30(13), 477-486.

Oliva, A. (2014). La conducta antisocial adolescente a la luz de las ciencias del cerebro. Revista de Psicología de Criança e do Adolescente, 4(1), 129-147.

Organización Mundial de la Salud. (2012). Prevención del suicidio SUPRE. http://www.who.int/mental_health/ prevention/suicide/suicideprevent/es/.

Organización Mundial de la Salud. (2014). Primer informe de la oms sobre prevención del suicidio. http://www.who. int/mediacentre/news/releases/2014/suicide-prevention-report/es/

Ortony, A. y Turner, T. J. (1990). What's Basic about Basic Emotions? Psychological Review, 97(3), 315.

Parra, D. y Tortosa, J. M. (2003). Violencia estructural: una ilustración del concepto. Documentación Social, 131(3), 57-72.

Páez, D., Martínez-Sánchez, F., Sevillano, V., Mendiburo, A. y Campos, M. (2012). Measurement of Affect Regulation Styles (MARS) Expanded to Anger and Sadness. Psicothema, 24(2), 249-254.

Pérez, I. (2010). Trastornos emocionales en la adolescencia. Revista Digital Transversalidad Educativa, 31. http:// www.enfoqueseducativos.es/transversalidad/transversalidad_31.pdf\#page=135

Peterson, A. L., Chen, J. I., Karver, M. S. y Labouliere, C. D. (2019). Frustration with Feeling: Latent Classes of Non-Suicidal Self-Injury and Emotion Regulation Difficulties. Psychiatry Research, 275(1), 61-70. https://doi. org/10.1016/j.psychres.2019.03.014

Pisani, P. A., Wyman, M., Petrova, K., Schmeelk-Cone, D. B., Goldston, Y. y Xia, M. S. (2013). Gould Emotion Regulation Difficulties, Youth-Adult Relationships, and Suicide Attempts among High School Students in Underserved Communities. Journal of Youth and Adolescence, 42(6), 807-820.

Rajappa, K., Gallagher, M. y Miranda, R. (2012). Emotion Dysregulation and Vulnerability to Suicidal Ideation and Attempts. Cognitive Therapy and Research, 36(6), 833-839.

República de Colombia. (1991). Constitución política de Colombia. Leyer.

Rodríguez, F. (2004). La pobreza como un proceso de violencia estructural. Revista de Ciencias Sociales (RCS), 10(1), $42-50$.

Schotte, D. E. y Clum, G. A. (1987). Problem-Solving Skills in Suicidal Psychiatric Patients. Journal of Consulting and Clinical Psychology, 55(1), 49.

Sen, A. (2008). Exclusión e inclusión. En A. Sen y B. Kliksberg (2008), Primero la gente. Una mirada desde la ética del desarrollo a los principales problemas del mundo globalizado (pp. 27-42). Planeta DeAgostini.

Shelef, L., Fruchter, E., Hassidim, A. y Zalsman, G. (2015). Emotional Regulation of Mental Pain as Moderator of Suicidal Ideation in Military Settings. European Psychiatry, 30(6), 765-769.

Sroufe, L. A. (2000). Desarrollo emocional: la organización de la vida emocional en los primeros años. Oxford University Press.

Skinner, E. A, Edge, K., Altman, J. y Sherwood, H. (2003). Searching for the Structure of Coping: A Review and Critique of Category Systems for Classifying Ways of Coping. Psychological Bulletin, 129(2), 216-269.

Suárez, Y. (2010). La inteligencia emocional como factor protector ante el suicidio. Duazary: Revista Internacional de Ciencias de la Salud, 7(2), 282-283.

Suárez, Y. (2011). La educación emocional como componente de la formación integral: una alternativa para la salud, el desarrollo humano y la calidad de vida. Duazary: Revista Internacional de Ciencias de la Salud, 8(2), 124-126.

Suárez, Y. (2012). La inteligencia emocional como factor protector ante el suicidio en adolescentes. Revista de Psicología GEPU, 3(1), 182-200. 
Suárez, Y. (2017). Factor socioeconómico, estilos de apego y regulación emocional en el riesgo e intento de suicidio en la adolescencia. Tesis de maestría. Universidad del Norte.

Subirats, J. (2004). Pobreza y exclusión social. Un análisis de la realidad española y europea. (Colección de Estudios 16). Fundación La Caixa. http://obrasocial.lacaixa.es/estudiossociales/estudiossociales_es.html

Teismann, T., Förtsch, E. M. A., Baumgart, P., Het, S. y Michalak, J. (2014). Influence of Violent Video Gaming on Determinants of the Acquired Capability for Suicide. Psychiatry Research, 215(1), 217-222.

Tezanos, J. F. (2001). La sociedad dividida. Estructuras de clase y desigualdades en las sociedades tecnológicas. Biblioteca Nueva.

Thompson, R. (1994). Emotion Regulation: A Theme in Search for Definition. Monographs of the Society for Research in Child Development, 59(2/3), 25-52.

Thompson, R. A. y Calkins, S. D. (1996). The Double-Edged Sword: Emotional Regulation for Children at Risk. Development and Psychopathology, 8(01), 163-182.

Thompson, R. A., Flood, M. F. y Lundquist, L. (1995). Emotional Regulation: Its Relations to Attachment and Developmental Psychopathology. En D. Cicchetti y S. L. Toth (eds.). Emotion, Cognition, and Representation. Rochester Symposium on Developmental Psychopathology. University of Rochester Press.

Thompson, R. A. y Goodman, M. (2010). Development of Emotional Regulation. En: A. M. Kring y D. M. Sloan, Emotion Regulation and Psychopathology: A Transdiagnostic Approach to Etiology and Treatment. The Guilford Press.

Kring, A. M. y Sloan, D. M. (2010). Development of Emotion Regulation. Emotion Regulation and Psychopathology: A Transdiagnostic Approach to Etiology and Treatment. The Guilford Press.

Victor, S. E. y Klonsky, E. D. (2014). Daily Emotion in Non- Suicidal Self- Injury. Journal of Clinical Psychology, 70(4), 364-375.

Van Orden, K. A., Witte, T. K., Cukrowicz, K. C., Braithwaite, S. R., Selby, E. A. y Joiner Jr., T. E. (2010). La teoría interpersonal del suicidio. Revisión Psicológica, 117(2), 575.

Voon, D., Hasking, P. y Martin, G. (2014). Change in Emotion Regulation Strategy Use and its Impact on Adolescent Nonsuicidal Self-Injury: A Three-Year Longitudinal Analysis using Latent Growth Modeling. Journal of Abnormal Psychology, 123(3), 487-498.

Werner, K. y Gross, J. J. (2010). Emotion Regulation and Psychopathology: A Conceptual Framework. En A. M. Kring y D. M. Sloan (eds.), Emotion Regulation and Psychopathology: A Transdiagnostic Approach to Etiology and Treatment (pp. 13-37). The Guilford Press.

Wolff, J. C., Thompson, E., Thomas, S. A., Nesi, J., Bettis, A. H., Ransford, B., Scopelliti, K., Frazier, E. A. y Liu, R. T. (2019). Emotion Dysregulation and Non-Suicidal Self-Injury: A Systematic Review and Meta-Analysis. European Psychiatry, 59(1), 25-36.

Zhao, X., Zhang, R. y Zheng, K. (2014). Gender Differences in Emotion Regulation Strategies in Adolescents. Chinese Journal of Clinical Psychology, 22(5), 849-854. 


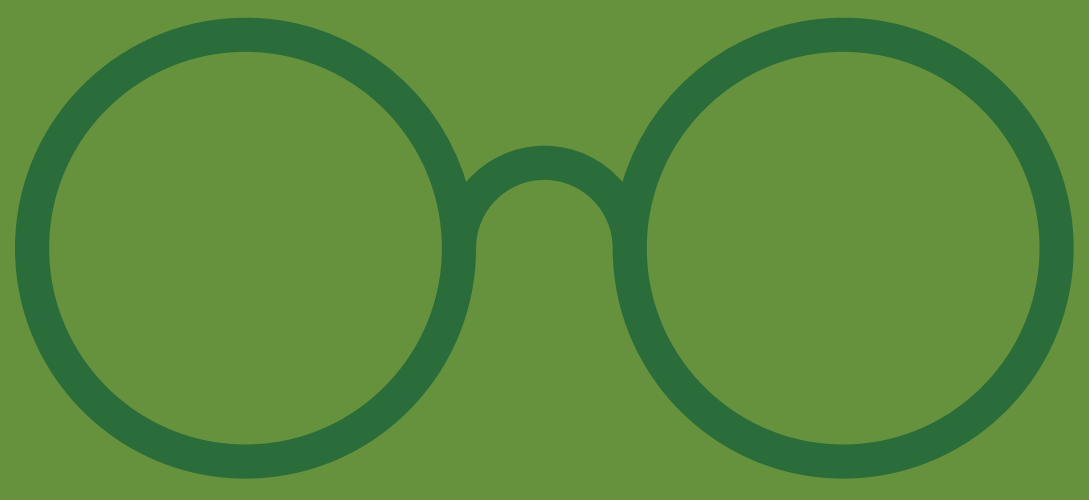

